\title{
A Crítica de Otto Klineberg aos testes de inteligência \\ O Brasil como laboratório racial
}

\section{Otto Klineberg's Critiques of Intelligence Testing}

Brazil as a Racial Laboratory

Marcos Chor Maio

Casa de Oswaldo Cruz

Fundação Oswaldo Cruz

Avenida Brasil 4036, Rio de Janeiro, RJ, 21.045-900, Brasil

maio@fiocruz.br

Resumo Otto Klineberg, professor de psicologia social na Universidade de Columbia, assegurou que os testes de inteligência eram uma ferramenta para legitimar hierarquias raciais. Ele conduziu numerosos estudos sobre as relações entre negros, brancos, índios e grupos de imigrantes nos Estados Unidos e na Europa na qual ressaltou que os aspectos ambientais superavam os fatores raciais. Klineberg concebia o Brasil como um ambiente privilegiado para demonstrar que os testes de inteligência não tinham qualquer base científica. $O$ presente artigo tem um foco triplo: três estudos realizados por Klineberg entre 1927 e 1935; suas experiências no Brasil de 1945 a 1947 como professor da Universidade de São Paulo, quando ele tentou investigar a relação entre testes de inteligência e condições socioeconômicas em escolas do Rio de Janeiro; e sua atuação inicial na Unesco, após a 2a Guerra Mundial, quando muitos ainda estavam convencidos que os testes de inteligência eram uma forma adequada para medir as chamadas habilidades inatas. Palavras-Chave testes de inteligência, racismo, Otto Klineberg

Recebido: 6 set. 2016 | Revisto pelo autor: 14 nov. 2016 | Aceito: 25 nov. 2016 http://dx.doi.org/10.1590/0104-87752017000100007

Varia Historia, Belo Horizonte, vol. 33, n. 61, p. 135-161, jan/abr 2017 
Abstract Otto Klineberg, professor of social psychology at Columbia University, held that intelligence tests were a tool for legitimizing racial hierarchies. He conducted numerous studies of the relations between blacks, whites, Indians, and immigrant groups in the United States and Europe in which he stressed that environmental considerations trumped racial factors. Klineberg came to see Brazil as a prime setting for demonstrating that intelligence testing lacked any scientific basis. The present article has a threefold focus: three studies conducted by Klineberg between 1927 and 1935; his experiences in Brazil from 1945 to 1947 as a professor at the University of São Paulo, where he tried to investigate the relation between intelligence testing and socioeconomic conditions at schools in Rio de Janeiro; and his post-World War II work at UNESCO, when many were still arguing that intelligence tests were an appropriate way to measure so-called innate traits.

KEYWORDs intelligence tests, racism, Otto Klineberg

\section{INTRODUÇÃO}

Psicólogo social e antropólogo canadense radicado nos EUA, Otto Klineberg (1899-1992) foi um crítico dos testes de inteligência como marcadores das diferenças raciais, enxergando-os como instrumento de legitimação de hierarquias socialmente constituídas. Klineberg empreendeu diversas pesquisas a fim de explorar as fragilidades dos testes, então em voga na Psicologia, e combater o racismo científico que serviu de fundamento, nos EUA, a políticas eugenistas. Seguindo os passos do antropólogo Franz Boas, seu antigo professor na Universidade de Columbia, Klineberg explorou as dimensões culturais e socioeconômicas do comportamento humano, assinalando o papel dos fatores ambientais no rol das explicações para as diferenças de desempenho demonstradas pelos diversos grupos constitutivos da sociedade norte-americana, dentre brancos, negros, indígenas e imigrantes.

Este artigo analisa a abordagem crítica desenvolvida por Klineberg aos testes de inteligência, enfocando seus trabalhos como professor da 
Universidade de Columbia no período do entreguerras, sua passagem pela Cadeira de Psicologia da Universidade de São Paulo (1945 a 1947) e o envolvimento nos debates que levaram à Primeira Declaração sobre Raça da Unesco no fim dos anos 1940. Klineberg concebeu o Brasil como terreno privilegiado para se testar a validade científica daqueles instrumentos de mensuração. Uma vez que o país, a seu ver, apresentava condições socioeconômicas mais favoráveis à população negra quando comparado ao quadro de desigualdades dos EUA, os resultados dos testes aplicados ao contexto brasileiro deveriam indicar menor discrepância entre negros e brancos, corroborando, deste modo, a tese acerca do peso da sociedade e da cultura no desenvolvimento da inteligência.

A primeira parte do artigo aborda três dos principais estudos de Klineberg realizados entre 1927 e 1935, quando aplica testes nos EUA e na Europa. Em seguida, o texto se atém à experiência brasileira de Klineberg e às suas tentativas de aplicar testes mentais em escolares do Rio de Janeiro estruturados em torno das variáveis raça e classe. Finalmente, o artigo se debruça sobre a atuação de Klineberg na Unesco, momento em que reemerge o debate sobre a validade dos testes de inteligência para a apreciação de alegadas características raciais inatas. O interesse de Klineberg pelos testes de inteligência persistiu após seus estudos dos anos 1930 e adentrou o contexto intelectual e político do pósguerra, o que sugere que não houve um cancelamento das concepções científicas racialistas depois dos eventos que marcaram as atrocidades nazistas.

\section{OS TESTES DE INTELIGÊNCIA}

Nascido em Quebec, Otto Klineberg (1899-1992) pertencia a uma tradicional família judaica de classe média oriunda do Império Austro -Húngaro que migrou para o Canadá na segunda metade do século XIX. Klineberg fez o bacharelado em Psicologia e Filosofia na Universidade McGill, mestrado em Filosofia na Universidade de Harvard, onde assistiu palestras sobre psicologia social ministradas por Floyd Allport. Retornou à Universidade McGill para fazer Medicina com a intenção 
de se tornar psiquiatra. Ao concluir o curso em 1925, decidiu ingressar no doutorado em Antropologia na Universidade de Columbia, após fazer uma disciplina curso de Linguística com o antropólogo Edward Sapir. Em Columbia, travou conhecimento com os psicólogos Robert S. Woodworth (1869-1962), Albert T. Poffenberger (1885-1977), Edward L. Thorndike (1874-1949) e Gardner Murphy (1895-1979) e assistiu às aulas de Edward Sapir (1884-1939), Ruth Benedict (1887-1948) e, principalmente, de seu orientador Franz Boas (1858-1942), que exerceu papel decisivo na construção de sua carreira acadêmica e profissional. Seus estudos se caracterizaram pelo cruzamento de temas caros à Antropologia, a exemplo da discussão do status científico do conceito de raça, e à Psicologia, como a validade dos testes de inteligência na aferição de diferenças raciais. Klineberg se definia como "a social psychologist with one foot in psychology and the other in cultural anthropology" (Klineberg, 1984, p.22; 1974).

Desde os anos 1920, quando realizou seu doutorado, Klineberg assume posição crítica face à crença social em hierarquias naturais entre as raças. A visão racialista adquirira respaldo científico nos EUA, sobretudo, com os testes de inteligência, que indicavam o melhor desempenho dos brancos quando comparados aos demais grupos raciais que constituíam a sociedade americana. ${ }^{1}$

Os testes remontam aos trabalhos desenvolvidos pelo eugenista britânico Francis Galton em fins do século XIX. Ao afirmar o caráter hereditário da capacidade intelectual humana, Galton propôs o uso de testes para a mensuração daquele atributo psicológico com base no pertencimento racial dos indivíduos, classificados a partir de dados antropométricos (Kevles, 1985, p.14-17; Carson, 2007, p.165-166). Os psicólogos franceses Alfred Binet (Universidade de Sorbonne) e Théodore Simon, que trabalhou em diversos hospitais na França, estiveram entre os primeiros a elaborar um instrumento de aferição da inteligência, a escala Binet-Simon, ao passo que os trabalhos do alemão Wilhelm Stern

1 Sobre Klineberg, ver DEGLER, 1991; RICHARDS, 1997; KEVLES, 1985. 
e do americano Lewis Terman difundiram o conceito de quociente de inteligência (Schneider, 1992; Kevles, 1985, p.79).

Nos EUA, entre as décadas de 1910 e 1920, os testes de inteligência ganharam relevo ao serem empregados por agências do Estado e organismos privados. Disseminou-se, entre os psicólogos, a crença de que a inteligência constituía um atributo psíquico fixo, quantificável e mensurável, e que de seu conhecimento científico dependia a reorganização racional da sociedade (Carson, 2007). Importante iniciativa neste sentido ocorreu durante a $1^{\text {a }}$ Guerra Mundial, quando as forças armadas se valeram do Army Alpha Test, ferramenta de avaliação desenvolvida pelos psicólogos Robert Yerkes (Harvard University), Henry Goddard (Ohio State University) e Lewis Terman (Stanford University), no intuito de selecionar recrutas (Gould, 1991). A partir de então, a aplicação de testes de inteligência se tornou uma das áreas de maior visibilidade em Psicologia, um dos seus principais recursos de legitimação científica e as técnicas de mensuração proliferaram em diversos setores, constituindo instrumento indispensável na classificação de indivíduos no sistema educacional e na produção industrial (Carson, 2007). Esse controle era o resultado de um amplo movimento migratório dos negros do Sul para o Norte dos EUA substituindo a mão de obra imigrante vinda da Europa Oriental e do Sul (Degler, 1991, p.197).

A aplicação dos testes em milhares de indivíduos durante a guerra gerou, pela primeira vez, dados em massa que estiveram na origem do livro A Study of American Intelligence (1923), influente estudo do psicólogo Carl Brigham, professor da Universidade de Princeton, que desempenhou papel-chave na consolidação das interpretações racialistas para a variação da capacidade mental. ${ }^{2}$ Interessado no estudo da associação entre hereditariedade e inteligência, Brigham estava convencido da superioridade cognitiva da raça branca ou "nórdica" frente aos demais grupos raciais que se faziam presentes na sociedade norte-americana (Kevles, 1985, p.82-83).

2 Brigham elaborou o Scholastic Aptitude Test (SAT), utilizado em processos de admissão escolar, a partir de uma reformulação do Army Alpha Test. GOULD, 1991. 
Ao sustentar que os estrangeiros representavam ameaça aos índices de inteligência do americano médio, a perspectiva de Brigham foi ao encontro das pressões oriundas das classes médias brancas por leis imigratórias mais rígidas, objeto de acirrados debates políticos nos EUA que redundaram no Immigration Restriction Act de 1924. Esta mesma visão racialista informou as políticas eugenistas de esterilização de deficientes. Considerados um modo objetivo de determinar as qualidades individuais, os testes de inteligência acabaram servindo à racionalização das práticas tradicionais de segregação e exclusão baseadas na etnicidade e no gênero (Carson, 2007, p.255). ${ }^{3}$

O trabalho de Brigham tornou-se uma referência obrigatória nos anos 1920 (Cravens, 1988, p.49-53), e não por acaso Otto Klineberg o elegeu como principal interlocutor em suas primeiras pesquisas. Buscando interpretações alternativas aos dados de Brigham, o psicólogo de Columbia ponderava que não era possível descartar a influência das condições socioeconômicas e das tradições culturais nas diferenças de desempenho demonstradas pelos grupos raciais submetidos aos testes. Klineberg, discípulo de Boas, questionava, ademais, a suposição de que a inteligência constituía um atributo biológico inato.

\section{AS PESQUisAS DE KLINEBERG: PONDO OS TESTES À PROVA}

A crítica aos pressupostos racialistas da Psicologia foi uma constante na produção intelectual de Klineberg entre 1920 e 1950 (Klineberg, 1944; 1951). Em sua tese de doutorado, An Experimental Study of Speed and Other Factors in "Racial" Differences (1928), Klineberg analisou o papel de fatores como educação e condição socioeconômica nas diferenças de desempenho demonstradas por brancos e não-brancos nos testes de inteligência (Klineberg, 1928, p.05).

3 Em meados da década de 1920, mais de oitenta por cento das cidades utilizavam testes de inteligência em escolas para classificar estudantes, especialmente no ensino elementar. CARSON, 2007, p. 255. 
Parte da pesquisa de campo foi realizada na reserva indígena de Yakima, Estado de Washington, em 1926. Klineberg aplicou seis testes da série Pintner-Paterson ${ }^{4}$ em 148 crianças indígenas e 107 crianças brancas que viviam na cidade de Toppenish, no coração da reserva, e cujas idades variavam entre 7 e 16 anos. Após verificar que o fator as crianças indígenas demoravam mais em responder às questões do que as brancas, Klineberg concluiu que o fator agilidade era dotado de valor somente em sociedades modernas e competitivas como a norte-americana, que a inculcavam desde cedo nas crianças, não podendo constituir critério absoluto de avaliação da inteligência: "a ênfase na velocidade [...] aparentemente não penetrou nos padrões de cultura indígenas, ao menos não na Reserva Yakima" (Klineberg, 1928, p.34-35).

Klineberg aplicou os mesmos testes em 200 meninos negros e 100 brancos, entre 11 e 16 anos, de escolas da cidade de Nova York. Após constatar que o índice de velocidade dos grupos de escolares negros aumentava proporcionalmente ao número de anos de residência em Nova York, Klineberg concluiu que havia "boas razões para acreditar [...] que a diferença no ambiente é de algum modo responsável por acelerar as atividades dos negros" (Klineberg, 1928, p.50). Diante dos resultados melhores obtidos pelos brancos, o psicólogo aventou a hipótese de que as condições ambientais a que brancos e negros estavam expostos não eram as mesmas, concentrando-se os últimos em bairros empobrecidos como o Harlem (Klineberg, 1928, p.51).

Klineberg deu continuidade a essas indagações ao aplicar, com auxílio do National Research Council e do Board of Fellowships in the Biological Sciences da Columbia University, uma série de testes em escolares de centros urbanos e rurais da França, Alemanha e Itália, ${ }^{5}$ publicando os resultados em A Study of Psychological Differences Between

4 Esta foi a primeira grande tentativa de desenvolver uma série padronizada de testes de desempenho. Os testes foram os seguintes: Mare and Foal Test, Casuist Form Board, Triangle Test, Healy Puzzle "A", Ship Test e Knox Cube Test.

5 Klineberg aplicou testes em escolas de pequenas comunidades rurais com menos de três mil habitantes e também de cidades como Paris, Hamburgo e Roma. KLINEBERG, 1931. 
"Racial" and National Groups in Europe (1931). Ao conceber a pesquisa, realizada entre 1927 e 1929, Klineberg tinha em mira os estudos sobre imigrantes que, na esteira do trabalho de Brigham, indicavam haver forte correlação entre inteligência e origem étnica. Para Klineberg, esta abordagem não demonstrava controle sobre as variáveis ambientais em jogo e com frequência incorria no baralhamento entre raça e nacionalidade (Klineberg, 1931, p.08).

A fim de comparar o desempenho dos alunos, de 10 a 12 anos, Klineberg agrupou-os em nórdicos, alpinos e mediterrâneos, classificação racial dos europeus presente no trabalho de Brigham. Valeu-se, neste caso, da Antropometria, medindo as dimensões do crânio e o índice cefálico das crianças (Klineberg, 1931, p.17). O psicólogo constatou que as populações dos países visitados eram heterogêneas do ponto de vista étnico, sendo difícil a identificação de tipos raciais puros. Notou, ademais, que a pontuação nos testes variava mais em função das regiões de origem das crianças do que de seu pertencimento racial. $O$ fato de amostras diversas de um mesmo grupo racial apresentarem resultados discrepantes colocava em xeque a força explicativa da categoria raça. A crença na superioridade mental dos nórdicos se mostrava igualmente problemática uma vez que a melhor ou pior colocação das raças na pontuação geral variava conforme o país (Klineberg, 1931, p.29-31). Dentre os seus achados, Klineberg destacou o melhor resultado obtido pelos meninos da cidade em comparação com os do campo, e concluiu que não seria "muito difícil encontrar uma explicação dos resultados em termos de diferença de formação e de ambiente" que considerasse o sistema escolar e a frequência de exposição a testes por parte das crianças (Klineberg, 1931, p.41).

De volta aos EUA, Klineberg empreendeu nova pesquisa, desta vez a partir do levantamento dos registros escolares de crianças negras em três cidades do Sul (Nashville, Birmingham e Charleston) mantidos ao longo de dez anos, e aplicou testes mentais em escolares negros de Nova Iorque, agrupando-os novamente de acordo com o tempo de residência na cidade. O estudo, publicado sob o título de Negro Intelligence and Selective Migration (1935), questionava em particular a hipótese da 
migração seletiva, segundo a qual os melhores resultados em testes da população negra urbana em comparação com os segmentos rurais podiam ser explicados pela capacidade dos negros de inteligência superior nascidos no Sul de fixar residência no Norte, constituindo sua migração um processo de seleção dos mais aptos (Klineberg, 1935, p.03).

Examinando os registros escolares, Klineberg constata que não havia diferenças significativas de desempenho entre as crianças negras que migravam para o Norte e a média das crianças negras das escolas sulistas das quais aquelas provinham. Ademais, as diferenças observadas entre as crianças oriundas das três cidades do Sul podiam ser creditadas a fatores econômicos e à elevação do grau de escolarização dos migrantes ocorrida nas primeiras décadas do século XX (Klineberg, 1935, p.23). Os resultados dos testes em Nova Iorque indicavam que o tempo de residência na cidade tinha efeito significativo sobre o desempenho das crianças negras provenientes do Sul rural (Klineberg, 1935, p.28). Contra a hipótese da migração seletiva, o psicólogo de Columbia concluía, mais uma vez, em favor das teses ambientais (Klineberg, 1935, p.59).

As objeções de Klineberg à validade dos testes levantadas a partir destas pesquisas foram veiculadas em obras como Race differences (1935), dedicada a Franz Boas, que sumariza os escritos psicológicos sobre o tema, ou Social Psychology (1940), de caráter mais geral e didático. Klineberg insiste nas limitações metodológicas dos testes, indicando a dificuldade em comparar amostras de grupos raciais distintos, tendo em vista a desigualdade das condições socioeconômicas em que viviam. Enquanto os negros ocupassem posições desvantajosas na sociedade norte-americana, não seria possível afirmar, em bases científicas, que seu baixo desempenho refletisse atributos raciais inatos. Os pesos relativos das variáveis ambientais e da variável raça no desenvolvimento da inteligência humana somente poderiam ser aquilatados em uma sociedade "em que os negros não sofram qualquer tipo de discriminação e desfrutem das mesmas oportunidades educacionais e econômicas [que os brancos]" (Klineberg, 1935, p.188). Ainda que fosse um lugar "difícil de achar", o Brasil, na perspectiva de Klineberg, estaria mais próximo desta realidade (Klineberg, 1935). 


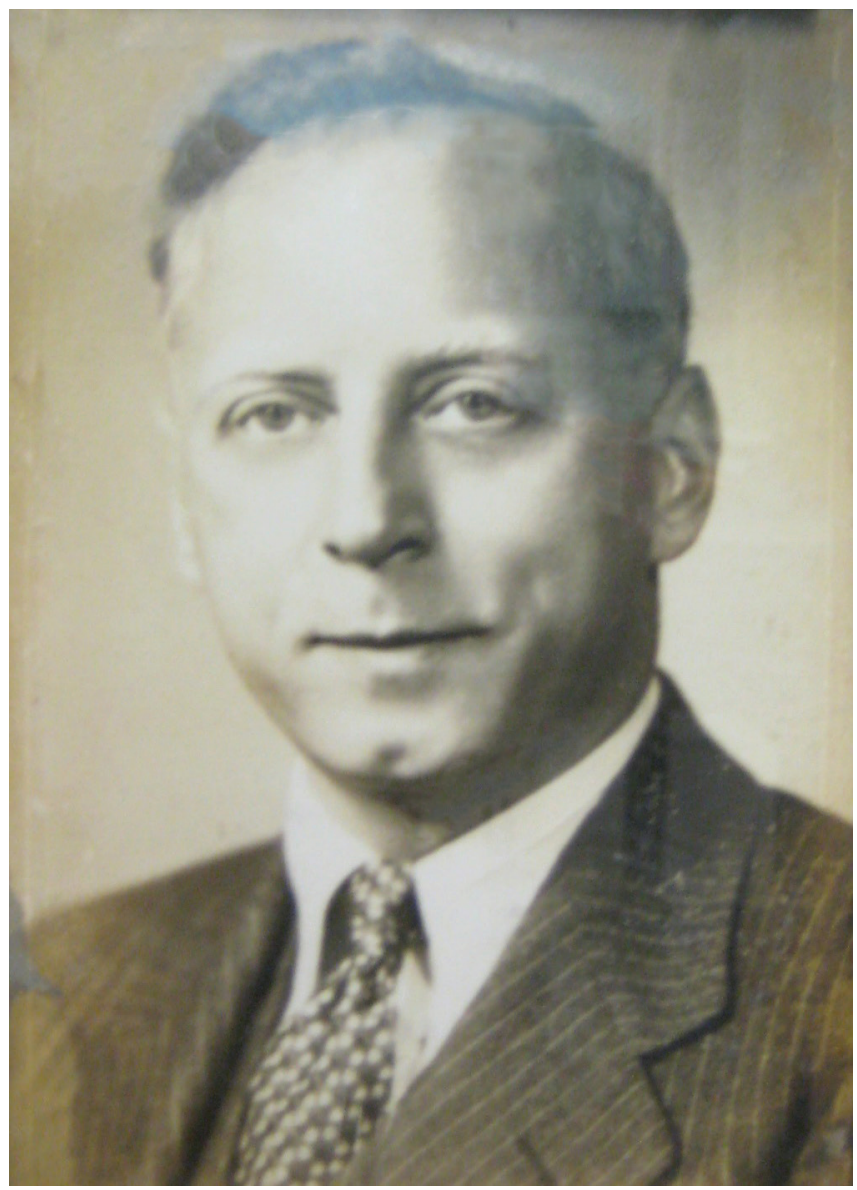

Foto: Otto-Klineberg, 1947 - Centro de Apoio à Pesquisa em História Sergio Buarque de Holanda - FFLCH - USP.

\section{O BRASIL COMO LABORATÓRIO}

Klineberg herdou de Franz Boas o interesse pelas relações raciais no Brasil. Em Anthropology and Modern Life (1928), livro de popularização da Antropologia que se inclui entre seus esforços de combate ao racismo, o antropólogo de Columbia havia chamado a atenção para as formas atenuadas de preconceito e discriminação existentes na sociedade brasileira 
relativamente à situação norte-americana, importante evidência contra a tese de que a aversão mútua constituía uma tendência natural das raças: "race feelings between Whites, Negroes and Indians in Brazil seems to be quite diferent from what it is among ourselves. On the coast there is a large Negro population. The admixture of Indian is also quite marked. The discrimination between these three races is very much less than it is among ourselves, and the social obstacles for the race mixture or for social advancement are not marked" (Boas, 1962 [1928], p.65).

A visão de Boas, por seu turno, foi inspirada pelo trabalho do historiador alemão Rudiger Bilden, que elaborou uma interpretação positiva da miscigenação no Brasil. No artigo Brazil: Laboratory of Civilization (1929), publicado em The Nation, prestigiosa revista norte-americana, ${ }^{6}$ Bilden destaca a importância da herança ibérica na formação social brasileira, especialmente no desenvolvimento de relações inter-étnicas propícias à miscigenação. Embora tenha indicado "certain amount of racial discrimination and friction", o historiador afirmou que os antagonismos existentes seguiam mais linhas de classe do que de raça, de modo que tendiam a desaparecer conforme as distinções sociais diminuíssem: "[...] broadly speaking race lines still follow class lines, despite generous overlapping. The lower the class, the darker the blood" (Bilden, 1929, p.71-72). Bilden critica intelectuais brasileiros como Euclides da Cunha e Oliveira Viana por conceberem os males do Brasil como resultado da miscigenação biológica da população e perderem de vista suas verdadeiras causas, de natureza sócio-histórica, que remontavam à dominação portuguesa nos trópicos. Ao qualificar o Brasil "as a world laboratory of tropical civilization" (Bilden, 1929, p.74), o artigo de Bilden condensa um imaginário social predominantemente harmonioso sobre o país partilhado por vários cientistas sociais norte-americanos nos 1930 e 1940, tais como: Robert Park, Franklin Frazier, Melville Herskovits, Donald Pierson, Lorenzo Turner e Ruth Landes. Tendo sempre como contraponto a experiência norte-americana, marcada pela segregação e pela

6 BILDEN, Rudiger. Brazil, laboratory of civilization. The Nation, n. CXXVIII, p.71-74, 1929. 
violência, o caso brasileiro foi avaliado como bem-sucedido no terreno das relações raciais (Palhares-Burke, 2013, p.183).

Klineberg reproduz este diagnóstico da situação racial brasileira, que percebe como menos desigual, em trabalhos publicados nos EUA ainda na década de 1930 (Klineberg, 1935; 1940). Além de poder preencher as lacunas das pesquisas sobre o nível mental dos diferentes grupos étnicos, o país servia de contraexemplo à crença acerca de indisposições inatas entre as raças: "there is very much less prejudice against the Negro in Brazil than in the United States, and race mixture is countenanced to an extent unheard of in our own country" (Klineberg, 1940, p.397).

Klineberg enxergou a possibilidade de testar aquelas hipóteses, enfocando os problemas da raça e das relações raciais, ao ser convidado para ministrar aulas na Universidade de São Paulo (USP). Entre 1945 e 1947, ele atuou como professor visitante da Faculdade de Filosofia, Ciências e Letras, tendo sido incumbido especialmente da tarefa de organizar o Departamento de Psicologia. O convite partiu do biólogo André Dreyfus, diretor da faculdade, que, no contexto da política de Boa Vizinhança do governo Roosevelt, visitara diversas instituições científicas e culturais nos EUA em uma viagem de seis meses patrocinada pelo Departamento de Estado norte-americano. Dreyfus tinha a missão de contratar cientistas daquele país para trabalharem na USP. O contato com Klineberg ocorreu por intermédio do geneticista Theodosius Dobzhansky, professor de Columbia (Klineberg, 1984, p.70-71). Dobzhansky ministrara aulas para Dreyfus em 1943, em um curso sobre evolução das espécies na USP, tendo iniciado, na ocasião, pesquisas em genética das populações examinando os cruzamentos de moscas drosófilas (Glick, 2008). ${ }^{7}$

Como outros cientistas a exemplo de Ashley Montagu, Ruth Benedict, Gene Weltfish, no entreguerras e no pós-2 ${ }^{\text {a }}$ Guerra, Dobzhansky publicara um livro de divulgação antirracista intitulado Heredity, Race

7 As pesquisas com as drosófilas tiveram continuidade quando Dobszhansky retornou ao Brasil em1948, organizando grupos de trabalho formados por biólogos brasileiros e estrangeiros. GLICK, 2008. 
and Society (1946) junto com o geneticista Leslie Dunn, seu colega em Columbia. O livro critica as falácias em torno do conceito de raça, como a suposta degeneração decorrente da miscigenação. Nas palavras de Dobzhansky e Dunn,

contrary to opinion vociferously expressed by some sincere but misguided people, ... a trend [toward race fusion] is not biologically dangerous. Mixing of closely related races may even lead to increased vigor. As for the most distantly separated races, there is no basis in fact to think that either biological stimulation or deterioration follows crossing. The widespread belief that human race hybrids are inferior to both of their parents and somehow constitutionally unbalanced must be counted among the superstitions (Dobzhansky; Dunn, 1946, p.114).

Klineberg estava afinado com as posições anti-racialistas de Dobzhansky e Dunn. Sua vinda para o país não apenas guardava relação com o projeto de realizar pesquisas em solo brasileiro como também indicava a crescente influência da tradição científica norte-americana sobre a USP (Marinho, 2001), instituição até então vinculada fortemente à tradição francesa no campo das Ciências Sociais (Massi, 1989). A viagem de Klineberg contou com o apoio do Departamento de Estado norte-americano, tendo sido negociada junto ao bibliotecário Archibald Macleish, Assistant Secretary of State for Cultural and Public Affairs. ${ }^{8}$

$\mathrm{Na}$ USP, Klineberg organizou cursos a partir dos diferentes temas e sub-áreas da Psicologia moderna, tal como esta era concebida nos EUA: Psicologia Geral, Escolas e Sistemas de Psicologia; Psicologia Social; Psicologia Diferencial; Psicologia da Personalidade e Psicologia Patológica. ${ }^{9} \mathrm{O}$ psicólogo conferia importância aos métodos quantitativo e experimental, elementos indispensáveis para a legitimidade científica da

8 Virá o Professor Otto Klineberg. Correio da Manhã (Rio de Janeiro), 08 abr. 1945, p.31.

9 Anuário da Faculdade de Filosofia, Ciências e Letras, Universidade de São Paulo (1939-1949). São Paulo: Seção de Publicações, vol. II, 1953, p.512. 
disciplina, e abordava os esforços crescentes em torno da mensuração da distribuição diferencial dos atributos psíquicos (Klineberg, 1946; 1953).

$\mathrm{Na}$ abordagem de Klineberg transparece o esforço de aproximação com as Ciências Sociais (Klineberg, 1953, p.132). Na visão boasiana do psicólogo, antes de tentarem fixar rigidamente as constantes do comportamento humano, os psicólogos deviam levar em consideração suas variações interculturais. ${ }^{10}$ Fenômenos como o comportamento emocional, a percepção, a memória e a capacidade intelectual, cujas bases orgânicas eram com frequência exploradas, deviam ser estudados à luz de influências culturais e sociais. Em matéria de relações raciais, a Psicologia devia enfocar os padrões de relacionamento entre os grupos raciais, privilegiando o estudo dos preconceitos, estereótipos e discriminações contra minorias, a exemplo da população negra nos EUA (Klineberg, 1953, p.150-155).

Klineberg renovou os cursos da Cadeira de Psicologia, organizou o curso de especialização em Psicologia da USP, dedicou-se à publicação de textos introdutórios à disciplina em Português e foi membro fundador da Sociedade de Psicologia de São Paulo, mobilizando, neste processo, uma extensa rede de psicólogos, educadores, psicanalistas e cientistas sociais em atuação no Brasil (Klineberg, 1946; 1953). Suas atividades contribuíram para o desenvolvimento, no país, de uma Psicologia de base empírica, processo que remonta aos esforços de modernização da educação brasileira que tiveram lugar nos anos 1920 e 1930. Sob o influxo de ideias pedagógicas em circulação nos EUA, intelectuais como Anísio Teixeira, Lourenço Filho, Noemy da Silveira e Isaías Alves, que estudaram no prestigioso Teachers College (Columbia University), enxergaram na aplicação de testes psicológicos a possibilidade de reorganizar o ensino público primário, então em lento processo de ampliação para as massas, a partir de

10 Klineberg nota que as fronteiras disciplinares entre Psicologia Social, Psicologia Individual, Sociologia e Antropologia eram difíceis de se manter na prática. Isto porque "[mesmo] isolado, o indivíduo mostra a influência do ambiente social e cultural no qual viveu” (KLINEBERG, 1946, p.10). 
critérios científico-racionais, isto é, levando em consideração o estudo das variações na capacidade de aprendizagem e inteligência de cada criança (Rocha, 2011). ${ }^{11}$

A agenda de pesquisas sobre raça e relações raciais que Klineberg procurou desenvolver no Brasil se evidencia a partir da correspondência mantida com o antropólogo Arthur Ramos, professor da Faculdade Nacional de Filosofia e estudioso da cultura afro-brasileira cujas pesquisas incorporavam a Psicanálise, a Psicologia Social e a Antropologia (Maio, 2015). Klineberg idealizou a aplicação de testes de inteligência entre escolares do Rio de Janeiro estruturados em torno das variáveis raça e classe assim como investigações acerca das atitudes relativamente aos diferentes grupos raciais: "My plan would be to conduct two major studies. The first on diferences in mental test results among school children classified according to 'race' (color) and economic class. The second on 'race relations', that is to say, patterns of friendliness and opposition between groups of diferente origin. I shall of course be glad to send you a detailed outline in advance if you wish" ${ }^{12} \mathrm{O}$ psicólogo revelou ainda a vontade de realizar estudos sobre estereótipos raciais, cotejando a forma como os negros eram retratados nas literaturas brasileira e norte-americana, e sobre o caráter nacional. ${ }^{13}$

11 DÁVILA, 2003, analisa as implicações dos testes para as políticas educacionais gestadas nos anos 1930 e os contornos racialistas que estas por vezes assumiram.

12 Carta de Otto Klineberg a Arthur Ramos, 31 mar. 1947, 1p., I-35,32,1,574. Arquivo Arthur Ramos, Biblioteca Nacional.

13 Em "Estudos Brasileiros", Roger Bastide analisa dois livros: Brasil: Uma interpretação (Gilberto Freyre) e Introdução à Antropologia Brasileira (Arthur Ramos). A certa altura do artigo, Bastide discorre sobre os planos de pesquisa que compartilhou com Otto Klineberg, mas que não foram adiante. Conforme Bastide: "Há outro estudo interessante que (...) o professor Klineberg e eu planejáramos realizá-lo juntos, mas não foi possível, cada um de nós ocupado com as próprias obrigações. Seria o estudo, em geral, dos estereótipos do negro, do índio e do estrangeiro, e dos conceitos populares sobre a questão, através da literatura, do jornalismo, etc. O elemento folclórico do problema já foi, em parte, abordado por Mário de Andrade no seu artigo 'O preconceito de cor', mas esse terreno é fecundo a pesquisas, ainda que já se fizeram estudos análogos nos Estados Unidos, por exemplo, no livro editado por Klineberg 'Characteristics of the American Negro', sendo possível, portanto, fazer-se uma comparação entre os dois resultados obtidos" (A Manhã (Rio de Janeiro), Letras \& Artes, 04 jan. 1948, 
Ao indicar seus interesses científicos, Klineberg reitera, em seus textos publicados no Brasil, a visão positiva que mantinha da situação racial do país. O psicólogo manifesta afinidades com a perspectiva de vários cientistas sociais que haviam se debruçado sobre o tema, tais como Gilberto Freyre, Arthur Ramos, Roger Bastide, Emilio Willems, Herbert Baldus e Donald Pierson, privilegiando os argumentos freyreanos acerca da herança ibérica, da miscigenação e da formação de um tipo de sociedade marcada pela harmonia (Klineberg, 1946, p.122).

A forma como Klineberg enxerga a sociedade brasileira se depreende ainda da conferência intitulada "Psicologia e caráter nacional", proferida na sessão inaugural da Sociedade de Psicologia de São Paulo, em dezembro de 1945. Abordando o problema do "caráter nacional", Klineberg indica a necessidade de substituição dos estudos apriorísticos, informados por estereótipos nacionais, por "provas objetivas" do comportamento e das atitudes comuns à maioria dos habitantes de cada país, o que era possível ser feito com base no instrumental técnico e metodológico à disposição da Psicologia e das Ciências Sociais (Klineberg, 1949, p.07). O caráter nacional refletia o "conjunto dos caracteres comuns a todo um povo" e podia ser explicado mediante o processo de socialização dos indivíduos de uma nacionalidade em padrões culturais próprios (Klineberg, 1949). ${ }^{14}$ Referindo-se ao Brasil, Klineberg observa que "um

p.03). Klineberg reitera seus interesses de pesquisa no Brasil em suas sugestões ao projeto Unesco de relações raciais (MAIO, 2001). Conferir: Comments on memorandum regarding Research on Race Relations in Brazil, p.06. Race Questions \& Protection of Minorities. REG 323.1. Part II up to 31/VII/50 (BOX REG 145), UNESCO archives.

14 O estudo do caráter nacional ganhou impulso entre psicólogos e cientistas sociais norte-americanos durante a $2^{\text {a }}$. Guerra Mundial, frente à necessidade de investigações estratégicas sobre os países envolvidos no conflito mundial. As primeiras pesquisas realizadas no período, ligadas aos estudos antropológicos de Cultura e Personalidade da Universidade de Columbia, foram levadas a cabo por Margaret Mead, Ruth Benedict, Gregory Bateson, Geoffrey Gorer, dentre outros, no âmbito do Committee for National Morale. Segundo NEIBURG; GOLDMAN, 1998, elas tenderam à reificação antropológica da categoria "nação" ao considerarem as fronteiras políticas construídas historicamente entre os países como fronteiras naturais que expressavam a existência de distintos universos socioculturais. 
país tão vasto e variado, feito de tantos elementos étnicos diferentes e praticando tão alto grau de democracia étnica, deveria constituir um excelente laboratório para pesquisa” (Klineberg, 1945, p.03). O "laboratório" seria um campo de experimentação, de interações raciais e culturais comumente concebidas em chave positiva e que inclui as ideias de mistura, de processo e de formação de um caráter nacional.

Nos textos de Klineberg transparece uma perspectiva otimista em relação à possibilidade de aplicação da Psicologia. Tendo em vista a construção do sistema das Nações Unidas no pós-2 ${ }^{\text {a }}$. Guerra, o conhecimento psicológico dos povos poderia ser empregado no estabelecimento de relações de cooperação internacional e no enfrentamento de problemas sociais e "fenômenos patológicos", como o preconceito racial (Klineberg, 1949, p.06; 1953, p.155).

As tentativas de Klineberg de desenvolver pesquisas em sua estada de dois anos na USP acabaram não se realizando em função, sobretudo, dos seus compromissos com o ensino, a pesquisa e a profissionalização da Psicologia em São Paulo. Contudo, seu interesse pelo estudo das relações raciais no Brasil, ao lado da visão crítica acerca dos testes de inteligência, persistiram nos anos subsequentes, em um contexto de crescente debate sobre o valor heurístico do conceito de raça no pós-2a Guerra Mundial, especialmente no âmbito da Unesco.

\section{RAÇA, TESTES E ENGAJAMENTO NOS DEBATES DA UNESCO}

Em setembro de 1947, Klineberg retornou à Universidade de Columbia e, em seguida, assumiu a direção do projeto Tensions Affecting International Understanding, iniciativa do Departamento de Ciências Sociais da Unesco que marcou os primórdios da organização. Aprovado na 2a sessão da Conferência Geral da Unesco, na cidade do México, em 1947, o “Tensions Project" tinha como meta o desenvolvimento de pesquisas na interface entre Antropologia Cultural e Psicologia Social, envolvendo Estudos de Cultura e Personalidade, caráter nacional, Ciências Sociais e Relações Internacionais. Entre os desafios propostos, encontrava-se 
o estudo das razões que levavam às guerras, às rivalidades nacionais e ao surgimento de estereótipos e preconceitos raciais contra minorias e imigrantes (Klineberg, 1950b)..$^{15}$

Os primórdios da Unesco refletiam o impacto causado pelo genocídio nazista. O principal objetivo da instituição era combater as tensões internacionais e assegurar a paz mundial promovendo o entendimento e a cooperação entre as nações. Não obstante a derrota do nazismo, o mundo emergiu profundamente abalado moral e intelectualmente. A superação da profunda crise da civilização ocidental exigia a recuperação na crença na razão liberal. A declaração de princípios contida no preâmbulo da Constituição refletia uma visão psicológica da 2a . Guerra Mundial: "Uma vez que as guerras nascem na mente dos homens, é na mente dos homens que as defesas da paz devem ser construídas". A agência internacional concebeu as doutrinas raciais que forneceram os fundamentos políticos -ideológicos da dominação hitlerista como um sistema antirracional de pensamento em confronto flagrante com as tradições humanistas inerentes à cultura ocidental. Afirmando que os conflitos e o ódio racial haviam sido fundados em postulados científicos falsos, a Unesco promoveu uma ampla campanha contra as bases ideológicas do racismo (Métraux, 1950).

Em abril de 1949, Klineberg alertava em conferência no Annual Meeting of the American Psychological Association para os perigos das "bombas atômicas, cortinas de ferro, alianças defensivas, chauvinismo, racismo, acusações e contra-acusações na Guerra Fria — tudo isso faz[endo] com que nos demos conta da realidade do perigo" (Klineberg, 1949, p.11). O psicólogo abordou de modo condensado uma série de iniciativas de pesquisa sobre conflitos internacionais no livro Tensions Affecting Internacional Understanding: a survey of research. $O$ Brasil emerge no capítulo "Influences Making For Aggression", em passagem que apresenta evidências de países com reduzida taxa de tensões raciais, experiências que deveriam ser consideradas na luta contra o racismo (Klineberg, 1950b, p.192).

15 Resolutions Adopted by The General Conference During its Second Session. México, nov./ dec. 1947. Paris, UNESCO, apr. 1948, p.25, UNESCO archives. 
Ainda no primeiro semestre de 1949 foi elaborado um plano educacional contra o racismo. Quando da divulgação da Declaração Universal dos Direitos Humanos, em dezembro de 1948, o Economic and Social Council (Ecosoc), órgão da Organização das Nações Unidas, propôs à Unesco a adoção de um programa de combate ao preconceito racial. Em setembro de 1949, a 4ª sessão da Conferência Geral da Unesco aprovou uma agenda de combate ao preconceito racial. Em seu programa de Ciências Sociais foi introduzido um capítulo especial, "Estudo e disseminação de fatos científicos concernentes a questões de raça”, no qual três objetivos se destacavam: 1) coletar e estudar materiais científicos referentes a questões raciais; 2) divulgar amplamente as informações científicas reunidas; 3) preparar uma campanha educacional baseada nessas informações. ${ }^{16}$

Três atividades foram realizadas. A primeira era a convocação de uma reunião de antropólogos (físicos e culturais) e sociólogos com o intuito de elaborar um manifesto científico a respeito do conceito de raça; a segunda se referia à realização de uma pesquisa sobre as relações raciais no Brasil $;{ }^{17}$ por último, a publicação de uma série de pequenos estudos nos campos da Biologia, da Genética, da Antropologia, da História e da Psicologia Social com o intuito de dar maior publicidade aos conhecimentos científicos a respeito dos temas raça e relações raciais (Dunn et al, 1972; Comas et al, 1970). ${ }^{18}$

16 Records of the General Conference of the United Nations Educational, Scientific and Cultural Organization, Fourth Session, Resolutions. Paris, Unesco, 1949, p.22, in Reg file 323.12 A 102. Part I (Box Reg 146), Arquivos da Unesco.

17 The Programme of Unesco proposed by the Executive Board, Part II - Draft Resolutions For 1951. Paris, 1950, p.40, Arquivos da Unesco. Sobre o debate acerca da Primeira Declaração Sobre Raça da Unesco e o projeto de pesquisa sobre as relações raciais no Brasil, ver MAIO, 1998.

18 Em 1949, Klineberg encaminhou à Comissão de Direitos Humanos do Ecosoc documento elaborado pela Unesco no qual constavam propostas de combate ao racismo. Encontram-se nele referências ao manifesto contra o nazismo elaborado pela Sociedade Brasileira de Antropologia e Etnologia, presidida por Arthur Ramos. Além disso, Klineberg sugeria que, como parte da campanha contra o racismo, era preciso ampliar a divulgação das obras de diversos cientistas sociais, entre os quais Gunnar Myrdal, Franz Boas, Ruth Benedict, Fernando Ortiz e Arthur Ramos. Economic and Social Council, United Nations, The Prevention of Discrimination and the Protection of Minorities, p.06, 09 maio 1949, in Race Questions \& Protection of Minorities. REG 323.1. Part I up to 30/VI/50 (box 145). Unesco Archives. 
Klineberg teve papel importante na implementação da agenda antirracista da Unesco. Para viabilizar a elaboração do manifesto científico acerca do conceito de raça, ele reuniu um conjunto de declarações contra o racismo publicadas por associações científicas, antes e durante a $2^{\text {a }}$. Guerra Mundial. Esse material deveria ser utilizado pelo grupo de especialistas "das diversas ciências sociais e biológicas" na elaboração do documento (Klineberg, 1949, p.20). O antropólogo Arthur Ramos, sucessor de Klineberg na direção do Departamento de Ciências Sociais da Unesco, foi o responsável por operacionalizar a proposta. Convidou cientistas liberais, socialistas e comunistas notoriamente antirracistas para a reunião de especialistas, que teve lugar em Paris entre os dias 12 e 14 de dezembro de $1949 .{ }^{19}$

Durante a Reunião de Experts, um dos pomos de discórdia, que colocou em posições opostas o antropólogo Ashley Montagu (Rutgers University), e o sociólogo Morris Ginsberg (London School of Economics), foi o entendimento sobre a relação entre comportamento e genética. Segundo Montagu, "de acordo com uma [então] recente pesquisa realizada nos EUA, era possível afirmar que nem as características mentais nem as comportamentais tinham qualquer significado genético". Contrapondo-se a Ginsberg, que acreditava que os testes de inteligência mensuravam variações nos níveis mentais dos indivíduos, Montagu, na tradição de psicólogos sociais norte-americanos liderada por Klineberg, considerava que "as condições econômicas, sociais e educacionais exerciam destacada influência sobre os resultados dos testes de inteligência". Ele também se posicionou criticamente quanto à associação entre aspectos físicos e mentais, afirmando que já havia sido "provado que as características de temperamento eram determinadas por fatores culturais". Montagu criticou, ademais, as visões negativas sobre a miscigenação e a tese segundo a qual "uma acentuada falta de

19 Fizeram parte da reunião os sociólogos Franklin Frazier (EUA), Morris Ginsberg (Inglaterra) e Luiz de Aguiar Costa Pinto (Brasil); os antropólogos Ernest Beaghole (Nova Zelândia), Juan Comas (México), Ashley Montagu (EUA) e Claude Lévi-Strauss (França); e o filósofo, educador e político Humayan Kabir (Índia). Sobre a Primeira Declaração sobre Raça da Unesco, ver MAIO; SANTOS, 2015; BARKAN, 1992; BRAITTAIN, 2007; HARRAWAY, 1989. 
harmonia resultava de alta percentagem de casos de cruzamentos de indivíduos com características étnicas muito diferentes". ${ }^{20}$

Em meio a discordâncias entre os experts, Montagu apresentou, na qualidade de relator, um rascunho da declaração que incluía pontos sensíveis ao debate, como a observação de que "os grupos humanos não [diferiam] em suas características mentais, incluindo a inteligência e o comportamento". O documento foi exposto ao escrutínio de 12 cientistas convidados a dar seus pareceres. ${ }^{21}$ Dentre as críticas que sofreu, destacaram-se aquelas dos geneticistas Leslie Dunn e Theodosius Dobzhansky e do próprio Otto Klineberg.

Leslie Dunn julgou imprecisa a afirmação que supunha não haver qualquer evidência quanto à existência de diferenças inatas entre grupos humanos. Dunn ponderou também que a negação de quaisquer conexões entre características morfológicas e mentais carecia de uma base de conhecimentos mais consistente, sendo necessário estabelecer uma diferenciação entre raça como fenômeno biológico e raça como mito. Segundo Dunn, "o mito consiste em se atribuir à raça poderes para os quais não há fundamentos biológicos".22

Próximo às ponderações de Dunn, Dobzhansky aconselhou a exclusão da seguinte frase: "Os fatos biológicos podem ser totalmente desconsiderados do ponto de vista do comportamento social e da ação social". ${ }^{23}$ Klineberg, por seu turno, julgava que a declaração deveria ser "menos dogmática". Afirmações definitivas quanto à ausência de

20 Economic and Social Council, United Nations, The Prevention of Discrimination and the Protection of Minorities, p.06, 9 maio 1949, in Race Questions \& Protection of Minorities. REG 323.1. Part I up to 30/VI/50 (box 145). Unesco Archives, p.3-5.

21 Os cientistas foram: Hadley Cantril (psicólogo social), E. G. Conklin (biólogo), Gunnar Dahlberg (geneticista), Theodosius Dobzhansky (geneticista), L.C.Dunn (geneticista), Donald Hager (antropólogo), Julien S. Huxley (biólogo), Otto Klineberg (psicólogo), Wilbert Moore (sociólogo), H.J.Muller (geneticista), Gunnar Myrdal (economista e sociólogo), Joseph Needham (bioquímico). MONTAGU, 1951, p.7-10.

22 Carta de Leslie Dunn a Robert Angell, 11 jan. 1950, p.1-2, in Reg file 323.12 A 102. Part I (Box Reg 146), Arquivos da Unesco.

23 Carta de Theodosius Dobzhansky a Robert Angell, 17 jan. 1950, p.02, in Reg file 323.12 A 102. Part I (Box Reg 146), Arquivos da Unesco. 
associação entre fatores biológicos e características psicológicas pareciam-lhe prematuras. Para o psicólogo, havia ainda certa precipitação em eliminar de vez os vínculos entre diferentes heranças genéticas e "realizações culturais". ${ }^{24}$

As críticas de Klineberg revelavam uma preocupação compartilhada por Dobzhansky, que sugeriu alterações em alguns enunciados que seriam "exageros e, por conseguinte, poderiam expor algumas das formulações ao ataque dos racialistas". ${ }^{25}$ Além de uma antecipação da crítica dos racialistas, as reservas de Klineberg quanto à declaração de Montagu podem ser compreendidas como denotando um ethos de homem de ciência que não tinha indicado um juízo definitivo sobre as correlações entre biologia e cultura, apesar dos seus diversos estudos críticos ao determinismo racial.

Ao fim de seis meses de consultas e debates internos, em julho de 1950, após a 5a sessão da Conferência Geral da Unesco. O conteúdo substantivo da declaração dos especialistas reunidos em Paris em 1949 não foi alterado. Todavia, os temores expressos por Dunn, Dobzhansky e Klineberg acabaram sendo levantados por outros pesquisadores após a divulgação oficial do documento pela Unesco. Ou seja, as críticas que se acumulavam nos círculos mais próximos passaram a ecoar amplamente, o que levou a Unesco a convocar uma nova reunião de especialistas que produziram a 2a . Declaração sobre Raça (Maio; Santos, 2015).

Pouco antes de lançar a Primeira Declaração sobre Raça, a 5 ${ }^{\text {a Confe- }}$ rência Geral da Unesco aprovou uma pesquisa sobre as relações raciais no Brasil. Subjacente à decisão de pesquisar um país latino-americano cuja população era considerada como produto da miscigenação (em suma, um país visto como uma espécie de experiência benigna em matéria racial), residia a expectativa de que o estudo poderia oferecer a prova científica definitiva para a refutação da existência de raças distintas.

24 Carta de Otto Klineberg a Robert Angell, 25 jan. 1950, p.01, in Reg file 323.12 A 102. Part I (Box Reg 146), Arquivos da Unesco.

25 Carta de Theodosius Dobzhansky a Robert Angell, 17 jan. 1950, p.01, in Reg file 323.12 A 102. Part I (Box Reg 146), Arquivos da Unesco. 


\section{CONSIDERAÇões FinaIS}

Desde os anos 1920, Klineberg vinha se posicionando criticamente frente à tese, então bastante difundida entre os psicólogos, da inferioridade racial de negros, índios norte-americanos e imigrantes com base nos resultados de testes de inteligência. Estava em consonância com Franz Boas, que tecera duras críticas à utilização dos testes, afirmando que o significado de seus resultados, com frequência tomados como indício de diferenças raciais inatas, não era auto-evidente (Boas, 1928, p.51-59). Além da tese de Klineberg sobre os índios Kashima, Boas havia orientado outras pesquisas sobre o tema, a exemplo da dissertação de mestrado da antropóloga Margaret Mead, Intelligence Tests of Italian and American Children (1924), que analisou o desempenho de filhos de imigrantes italianos nos testes de inteligência a partir de suas condições sociais e do tempo de sua residência nos Estados Unidos (Mead, 1927).

Em diversos momentos de suas críticas aos testes de inteligência, expressas em pesquisas, artigos e livros, Klineberg se referiu ao Brasil como um país que permitiria aferir da forma mais fidedigna a consistência daquelas ferramentas de avaliação psicológica. Em meados dos anos 1940, o psicólogo acreditava que o país constituía uma "democracia étnica", de tal modo que negros e brancos estariam sujeitos a condições ambientais relativamente semelhantes. Aos olhos de Klineberg, a situação racial brasileira, ao diferir do quadro de profundas desigualdades educacionais e econômicas entre negros e brancos existente nos EUA, poderia servir de importante laboratório para a aferição da capacidade intelectual de indivíduos de ambos os grupos raciais uma vez que a influência da variável raça poderia ser isolada na aplicação dos testes e devidamente apreciada. O Brasil representava a possibilidade de se buscar evidências para a interpelação do racismo nos EUA, estratégia comumente utilizada por diversos intelectuais entre os anos 1920 e 1960.

A agenda de pesquisas que Klineberg delineou durante sua estada no Brasil indica que a controvérsia acerca dos testes e do peso relativo da variável raça no desenvolvimento da inteligência ainda interessava fortemente ao psicólogo de Columbia naquele momento - questão que 
não teria sido resolvida nem mesmo posteriormente, como relevam os debates na Unesco em torno da Primeira Declaração sobre Raça.

A persistência do valor atribuído aos testes de inteligência mostrou-se vigoroso na discussão que resultou na $2^{\text {a }}$. Declaração sobre Raça, como se verifica em um parágrafo do documento: "Most anthropologists do not include mental characteristics in their classification of human races. Studies within a single race have shown that both innate capacity and environmental opportunity determine the results of texts of intelligence and temperament...." ${ }^{26}$ A citação representa uma crítica as posições de Klineberg ao retomar de modo afirmativo a importância das características inatas na formação psicossocial dos indivíduos. Como resposta, Klineberg (1950a) permaneceu afirmando, em perspectiva boasiana, o primado da cultura sobre a natureza em diversas publicações.

\section{Agradecimentos}

Agradeço à Renata Prudêncio, pelo auxílio no trabalho de pesquisa para o artigo, as atentas observações de Thiago Lopes e os valiosos comentários, críticas e sugestões dos pareceristas anônimos. Este artigo faz parte de uma pesquisa que conta com o apoio do CNPq.

\section{REFERÊNCIAS BIBLIOGRÁFICAS}

BARKAN, Elazar. The retreat of scientific racism. Cambridge: Cambridge University Press, 1992.

BOAS, Franz. Anthropology and Modern Life. New York: W. W. Norton, 1962 [1928].

BRATTAIN, Michelle. Race, racism, and antiracism: UNESCO and the politics of presenting science to the postwar public. American Historical Review, vol. 112, n. 5, p.1386-1413, 2007.

26 UNESCO. The Concept of Race: Results of an Inquiry. Paris: UNESCO, 1952, p.13. 
CARSON, John. The measure of merit: talents, intelligence, and inequality in the french and american republics, 1750-1940. Princeton: Princeton University Press, 2007.

COMAS, Juan et al.. Raça e Ciência, vol. I. São Paulo: Editora Perspectiva, 1970 [1960].

CRAVENS, Hamilton. The Triumph of Evolution: The Heredity-Enviroment Controversy, 1900-1941. Baltimore: The Johns Hopkins University, 1988. DÁVILA, Jerry. Diploma of Whiteness: race and social policy in Brazil, 1917 - 1945. Durham and London: Duke University Press, 2003.

DEGLER, Carl N. In search of human nature: the decline and revival of Darwinism in American Social Thought. New York, Oxford: Oxford University Press, 1991.

DOBZHANSKY, Theodosius; DUNN, Leslie. Heredety, Race and Society. New York: Penguin, Harmondsworth, 1946.

DUNN, Leslie et al.. Raça e Ciência vol. II. São Paulo: Editora Perspectiva, 1972 [1960].

GLICK, Thomas F. O programa brasileiro de genética evolucionária de populações, de Theodosius Dobzhansky. Revista Brasileira de História, vol. 28, n. 56, p.315-325, 2008.

GOULD, Stephen Jay. A falsa medida do homem. São Paulo: Martins Fontes, 1991.

HARRAWAY, Donna. Primate Visions: Gender, Race and Nature in the World of Modern Science. New York: Routledge, 1989.

KEVLES, Daniel J. In the name of eugenics: genetics and the uses of human heredity. Cambridge: Harvard University Press, 1985.

KLINEBERG, Otto. A study of psychological differences between racial and national groups in Europe. Archives of Psychology, n. 132, p.1-58, sept. 1931.

KLINEBERG, Otto. An Experimental Study of Speed and Other Factors in 'Racial' Differences. Archives of Psychology, n. 15, p.5-111, jan. 1928.

KLINEBERG, Otto (org.). Characteristics of the American Negro. New York and Evanston: Harper \& Brothers, 1944.

KLINEBERG, Otto. Interview with Otto Klineberg. Columbia oral history series. Interviewer: Robert Rieber. John Jay College: CUNY, 1984. p.1-123 
KLINEBERG, Otto. Introdução à Psicologia Social. Boletim da Cadeira de Psicologia da Universidade de São Paulo, n. 1, p.1-139, 1946.

KLINEBERG, Otto. Létude scientifique des stéréotypes nationaux. Bulletin International des Sciences Sociales, vol. III, n. 3, p.546-556, 1951.

KLINEBERG, Otto. Otto Klineberg. In: LINDZEY, Gardner (org.). A History of Psychological in Autobiography, vol. VI. Prentice-Hall, Inc., Englewood Cliffs, New Jersey: Harvard University, 1974. p.161-182.

KLINEBERG, Otto. Psicologia e Caráter Nacional. Boletim da Sociedade de Psicologia de São Paulo, ano I, n. 1, p.03, dez. 1945.

KLINEBERG, Otto. Psicologia e Caráter Nacional. Boletim de Psicologia, ano I, n. 1, p.6-17, 1949.

KLINEBERG, Otto. Psicologia social. In: KLINEBERG, Otto (org.). A Psicologia Moderna. São Paulo: Editora Agir, 1953. p.132-156.

KLINEBERG, Otto. Race Differences. New York and London: Harper and Brothers, 1935.

KLINEBERG, Otto. Race Differences: the Present Position of the Problem. International Social Sciences Bulletin, vol. II, n. 4, p.460-466, 1950a.

KLINEBERG, Otto. Social Psychology. New York: Henry Holt and Company, 1940.

KLINEBERG, Otto. Tensions Affecting International Understanding. New York: Social Science Research Council, 1950b.

MAIO, Marcos Chor. Caminhos de Arthur Ramos: a busca do Brasil como projeto civilizatório. In: HOCHMAN, Gilberto; LIMA, Nísia Trindade (orgs.). Médicos Intérpretes do Brasil. São Paulo: Editora Hucitec, 2015. p.362-389.

MAIO, Marcos Chor. O Brasil no concerto das nações: a luta contra o racismo nos primórdios da UNESCO. História, Ciências, Saúde-Manguinhos, vol. 5, n. 2, p.375-413, 1998.

MAIO, Marcos Chor. UNESCO and the study of race relations in Brazil: National or regional Issue?. Latin American Research Review, vol. 36, n. 2, p.118-136, 2001.

MAIO, Marcos; SANTOS, Ricardo Ventura. Anti-racism and the uses of science in the post-World War II: An analysis of UNESCO's first statements on race (1950 and 1951). Vibrant, vol. 12, n. 2, p.1-25, jul./dez. 2015. 
MARINHO, Maria Gabriela Silva Martins da Cunha. Norte-americanos no Brasil: uma história da Fundação Rockefeller na Universidade de São Paulo (1932-1952). São Paulo: Editora Autores Associados/Fapesp/ USF, 2001.

MASSI, Fernanda Peixoto. Franceses e Norte-americanos nas Ciências Sociais Brasileiras (1930-1960). In: MICELI, Sérgio (org.). História das Ciências Sociais no Brasil, vol. 1. São Paulo: Idesp/Vértice/Finep, 1989. p.410-460.

MEAD, Margaret. Group intelligence tests and linguistic disability among Italian children. School \& Society, vol. 25, p.465-468, 1927.

MÉTRAUX, Alfred. UNESCO and the racial problem. International Social Science Bulletin, vol. II, n. 3, p.384-390, 1950.

MONTAGU, Ashley. Statement on race. New York: Henry Schuman, 1951. NEIBURG, Federico; GOLDMAN, Marcio. Antropology and Politics in Studies of National Character. Cultural Anthropology, vol. 13, n. 1, p.56-81, fev. 1998.

PALHARES-BURKE, Maria Lucia. Brasil, Laboratório de Civilização: a importância de Rudinger Bilden. Política \& Trabalho, n. 39, p.179-194, out. 2013.

RICHARDS, Graham. Race, racism and psychology: towards a reflexive history. London, New York: Routledge, 1997.

ROCHA, Ana Cristina Santos Matos. O que fazer com os rudes?: Isaías Alves e as divergências sobre o papel da inteligência na organização escolar (1930-1942). Dissertação (Mestrado em História) - CPDOC- Fundação Getúlio Vargas. Rio de Janeiro, 2011.

SCHNEIDER, William H.. After Binet: French Intelligence Testing, 1900-1950. Journal of The History of the Behavioral Sciences, vol. 28, p.111-132, apr. 1992. 